\title{
Osteotomies around the knee lead to corresponding frontal realignment of the ankle
}

\author{
Christian Konrads $^{1} \cdot$ Alexander Eis $^{1} \cdot$ Sufian S. Ahmad ${ }^{1,2} \cdot$ Ulrich Stöckle $^{2} \cdot$ Stefan Döbele $^{1}$
}

Received: 22 March 2021 / Accepted: 21 May 2021 / Published online: 4 June 2021

(c) The Author(s) 2021

\begin{abstract}
Introduction Despite the fact that osteotomies around the knee represent well-established treatment options for the redistribution of loads and forces within and around the knee joint, unforeseen effects of these osteotomies on the ankle are still to be better understood. It was therefore the aim of this study to determine the influence of osteotomies around the knee on the coronal alignment of the ankle. We hypothesize that osteotomies around the knee for correction of genu varum or valgum lead to a change of the ankle orientation in the frontal plane by valgisation or varisation.

Materials and methods Long-leg standing radiographs of 154 consecutive patients undergoing valgisation or varisation osteotomy around the knee in 2017 were obtained and utilized for the purpose of this study. Postoperative radiographs were obtained after union at the osteotomy site. The hip knee ankle angle (HKA), the mechanical lateral distal femur angle (mLDFA), the mechanical medial proximal tibia angle (mMPTA) and five angles around the ankle were measured. Comparison between means was performed using the Wilcoxon-Mann-Whitney test.

Results One hundred fifty-four patients (96 males, 58 females) underwent osteotomies around the knee for coronal realignment. The mean age was $51 \pm 11$ years. Correction osteotomies consisted of 73 HTO, 54 DFOs, and 27 double level osteotomies. Of all osteotomies, 118 were for valgisation and 36 for varisation. For valgisation osteotomies, the mean HKA changed from $5.8^{\circ} \pm 2.9^{\circ}$ preoperatively to $-0.9^{\circ} \pm 2.5^{\circ}$ postoperatively, whereas the mMPTA changed from $85.9^{\circ} \pm 2.7^{\circ}$ to $90.7^{\circ} \pm 3.1^{\circ}$ and the malleolar-horizontal-orientation-angle (MHA) changed from $16.4^{\circ} \pm 4.2^{\circ}$ to $10.9^{\circ} \pm 4.2^{\circ}$. For varisation osteotomies, the mean HKA changed from $-4.3^{\circ} \pm 3.7^{\circ}$ to $1.1^{\circ} \pm 2.2^{\circ}$ postoperatively, whereas the mLDFA changed from $85.7^{\circ} \pm 2.2^{\circ}$ to $89.3^{\circ} \pm 2.3^{\circ}$ and the MHA changed from $8.8^{\circ} \pm 5.1^{\circ}$ to $11.2^{\circ} \pm 3.2^{\circ}$.

Conclusion Osteotomies around the knee for correction of coronal limb alignment not only lead to lateralization or medialization of the weight-bearing line at the knee but also lead to a coronal reorientation of the ankle. This can be measured at the ankle using the MHA. When planning an osteotomy around the knee for correction of genu varum or valgum, the ankle should also be appreciated — especially in patients with preexisting deformities, ligament instabilities, or joint degeneration around the ankle.
\end{abstract}

Keywords Deformity $\cdot$ Osteotomies $\cdot$ Realignment $\cdot$ Valgisation $\cdot$ Varisation

\section{Abbreviations}

aLDTA Anatomic Lateral Distal Tibia Angle

AMA Anatomic Mechanical Angle of the femur

HKA Hip Knee Ankle angle

JLCA Joint line conversion angle

mTFA Mechanical tibio-femoral angle

Christian Konrads

christian.konrads@gmail.com

1 Department for Trauma and Reconstructive Surgery, BG Klinik, University of Tübingen, Tübingen, Germany

2 Center for Musculoskeletal Surgery, Charité - University Medical Center Berlin, Berlin, Germany
mLDFA Mechanical Lateral Distal Femoral Angle

mLPFA Mechanical Lateral Proximal Femoral Angle

mMPTA Mechanical Medial Proximal Tibial Angle

aLDTA Anatomic Lateral Distal Tibia Angle

mLDTA Mechanical Lateral Distal Tibia Angle

mMA Mechanical Malleolar Angle

MHA Malleolar Horizontal Orientation Angle

SD Standard Deviation

TPHA Tibia Plafond Horizontal Orientation Angle

TTTA Tibio Talar Tilt Angle 


\section{Introduction}

Osteotomies around the knee represent powerful modalities for the treatment of bony deformities and degenerative joint disease [1-4]. The intended effects of these osteotomies act on joints by redistributing loads and force vectors $[5,6]$. Despite the fact that osteotomies around the knee represent well-established treatment options for the redistribution of loads and forces within and around the knee joint, unforeseen effects of these osteotomies on the ankle are still to be better understood. Although osteotomies around the knee are successful orthopaedic standard procedures, it is not known to what extent coronal ankle alignment might be intentionally or unintentionally altered.

It was therefore the aim of this study to determine the influence of osteotomies around the knee on the coronal alignment of the ankle. We hypothesize that osteotomies around the knee for correction of genu varum or valgum lead to a change of the ankle orientation in the frontal plane by valgisation or varisation. This new knowledge would help to treat patient better by improving the planning of osteotomies and avoiding unwanted effects on the adjacent ankle joint.

\section{Patients and methods}

The patient cohort included 154 knees of 154 patients undergoing osteotomies around the knee due to bony malalignment and corresponding symptoms. The mean age was $51 \pm 11$ years. There were 96 male and 58 female patients. All osteotomies performed were around the knee and included 73 high tibial osteotomies (HTO), 54 distal femur osteotomies (DFO) and 27 double level osteotomies. Of all osteotomies, 118 were valgisation osteotomies and 36 were varisation osteotomies. All consecutive patients were treated in a single center in the year 2017. Patients were excluded, if a multiple plane correction was performed, no magnification device was present on the postoperative radiograph, or image quality was inferior. Considering the above criteria, 154 knees of 154 patients undergoing osteotomy were considered eligible for retrospective data retrieval and inclusion in the study (Fig. 1). Ethical approval was received for the conduction of this study (421/2020BO).

All osteotomies were planned using a landmark based deformity analysis [7, 8]. A high tibial osteotomy was performed as described by Staubli and Lobenhoffer using a TomoFix MHT plate fixator (DePuy Synthes, Solothurn, Switzerland) [9-11]. Distal femoral osteotomy was performed using a medial subvastus approach and the technique described by Lobenhoffer [12-14]. For fixation, a TomoFix MDF plate (DePuy Synthes, Solothurn, Switzerland)

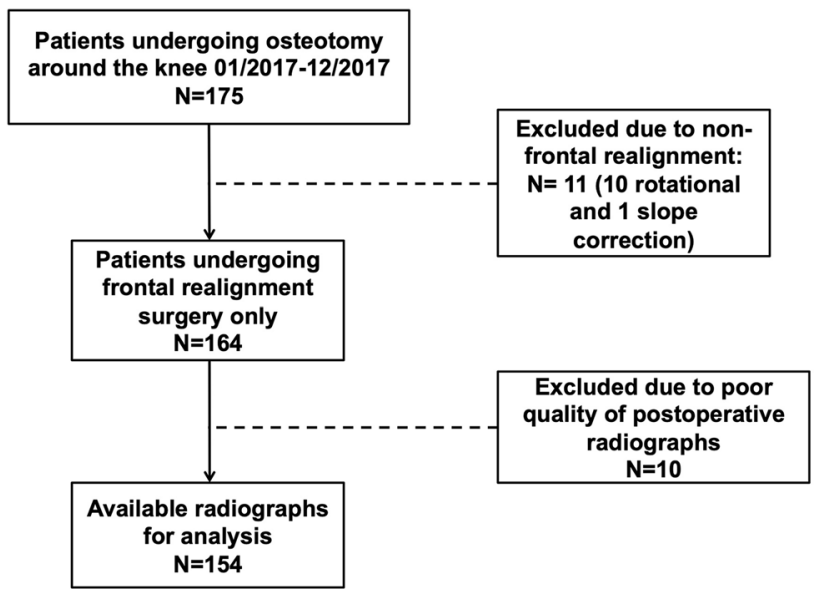

Fig. 1 Flowchart demonstrating inclusion

was used [15]. Double level osteotomy was performed as described by Schröter et al. [16].

Long-leg weight-bearing radiographs were obtained in accordance with Paley using a $1.3 \mathrm{~m}$ cassette (Global Imaging Baltimore, MD) [7]. Long leg antero-posterior standing radiographs were obtained with the patient standing in a bipedal stance in front of the long film cassette. The radiography tube was positioned in a distance of $305 \mathrm{~cm}$. The selected film cassette was of sufficient length to include the hips, knees, and ankles. The magnification with this setup was approximately $5 \%$. A calibration device $(250 \mathrm{~mm}$ steel ball) was used to calibrate the radiographs. The $\mathrm{X}$-ray beam was centered on the level of the knee joints.

Radiologic technical assistants were instructed to position both legs with the patella centered between the femoral condyles. It was of ultimate importance to ensure a standardized radiography.

Preoperative radiographs were obtained prior to surgery for planning of the deformity correction and were repeated postoperatively after union at the osteotomy site and recovery of limp-free full weight-bearing (Fig. 2).

Radiographic parameters were determined with an accuracy of $0.1 \mathrm{~mm}$ using mediCAD ${ }^{\circledR}$ (Hectec, Landshut, Germany). The following parameters were assessed in accordance to Paley [7]:

- Mechanical medial proximal tibial angle (mMPTA)

- Mechanical lateral distal femoral angle (mLDFA)

- Mechanical lateral proximal femoral angle (mLPFA)

- Anatomic Mechanical Angle of the femur (AMA)

- Hip Knee Ankle (HKA) angle, refers to the angle between mechanic axes of the femur and the tibia (Fig. 3). A synonym for HKA is the mechanical tibio-femoral angle (mTFA). 


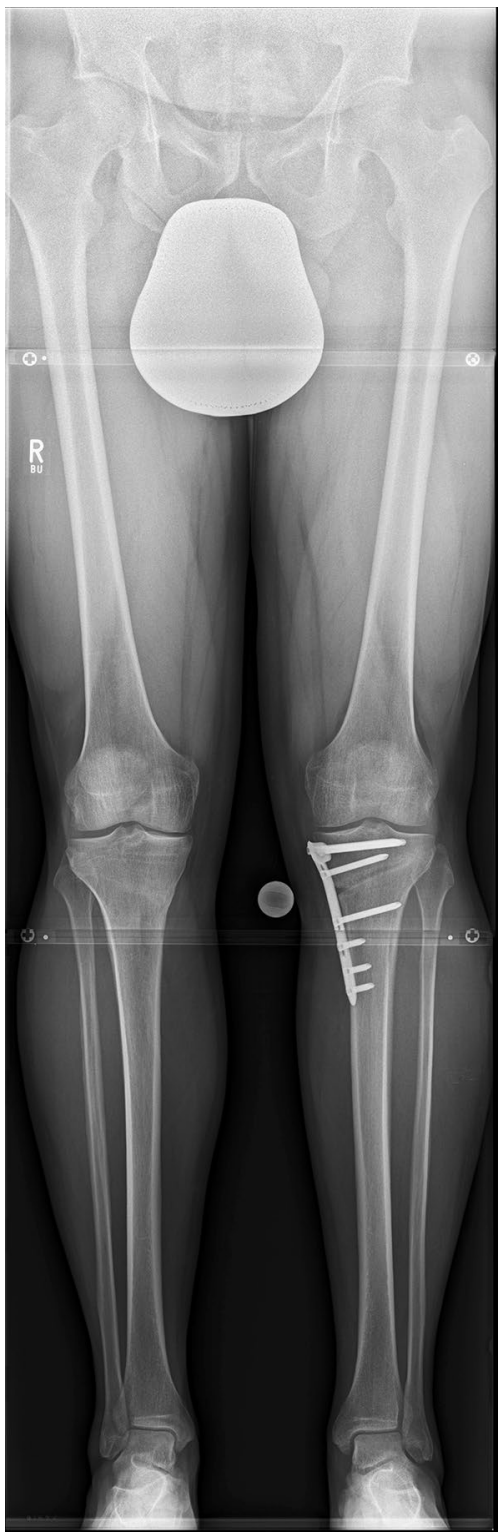

Fig. 2 Anteroposterior long-leg weight-bearing radiograph after high tibial osteotomy for valgisation of genu varum

At the level of the ankle, we measured the following radiographic parameters (Fig. 4):

- Mechanical Lateral Distal Tibia Angle (mLDTA)

- Mechanical Malleolar Angle (mMA)

- Malleolar Horizontal Orientation Angle (MHA)

- Tibia Plafond Horizontal Orientation Angle (TPHA)

- Tibio Talar Tilt Angle (TTTA)

For TPHA and TTTA, we defined positive values $>0^{\circ}$ to be varus and negative values $<0^{\circ}$ to be valgus.

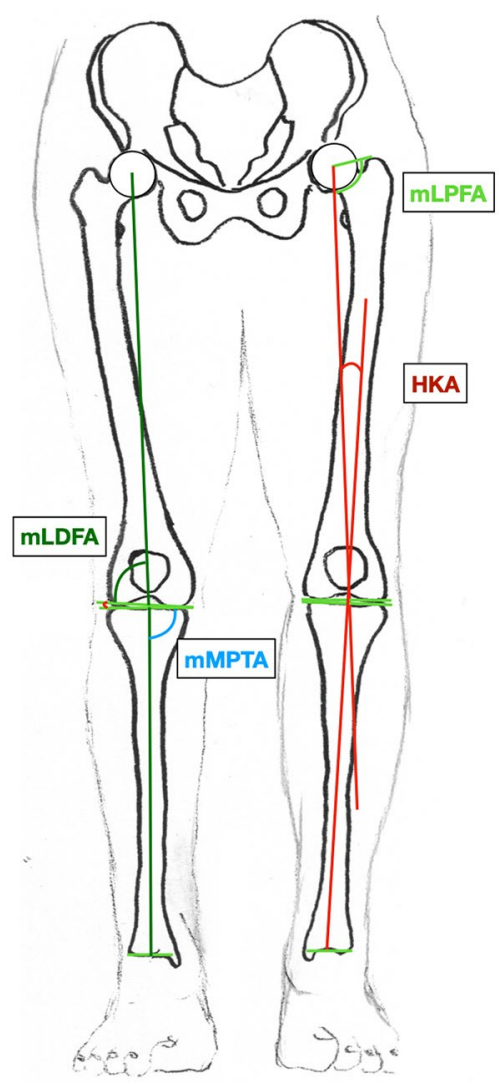

Fig. 3 Illustration of the radiographic parameters measured on a long-leg standing X-ray with the knees pointing forward. Measures around the hip and the knee, HKA, Hip Knee Ankle angle, mLDFA, Mechanical lateral distal femoral angle; mLPFA, Mechanical lateral proximal femoral angle, mMPTA, Mechanical medial proximal tibial angle

\section{Statistical analysis}

Continuous variables were presented as mean \pm standard deviation or range. Comparison between means was performed using the Wilcoxon test. A $p$ value of $<0.05$ was considered statistically significant. SPSS version 24 (IBM, Armonk, NY, USA) was used. A posthoc analysis was performed to ensure sufficient power to address the primary research question. Given the sample size of 154 patients, an effect size of 1.7 and an alpha error of 0.05 , the power of the study was calculated to be $95 \%$.

\section{Results}

Valgisation or varisation osteotomies around the knee led to significant changes regarding the coronal limb alignmentnot only around the knee but also at the ankle. This was 

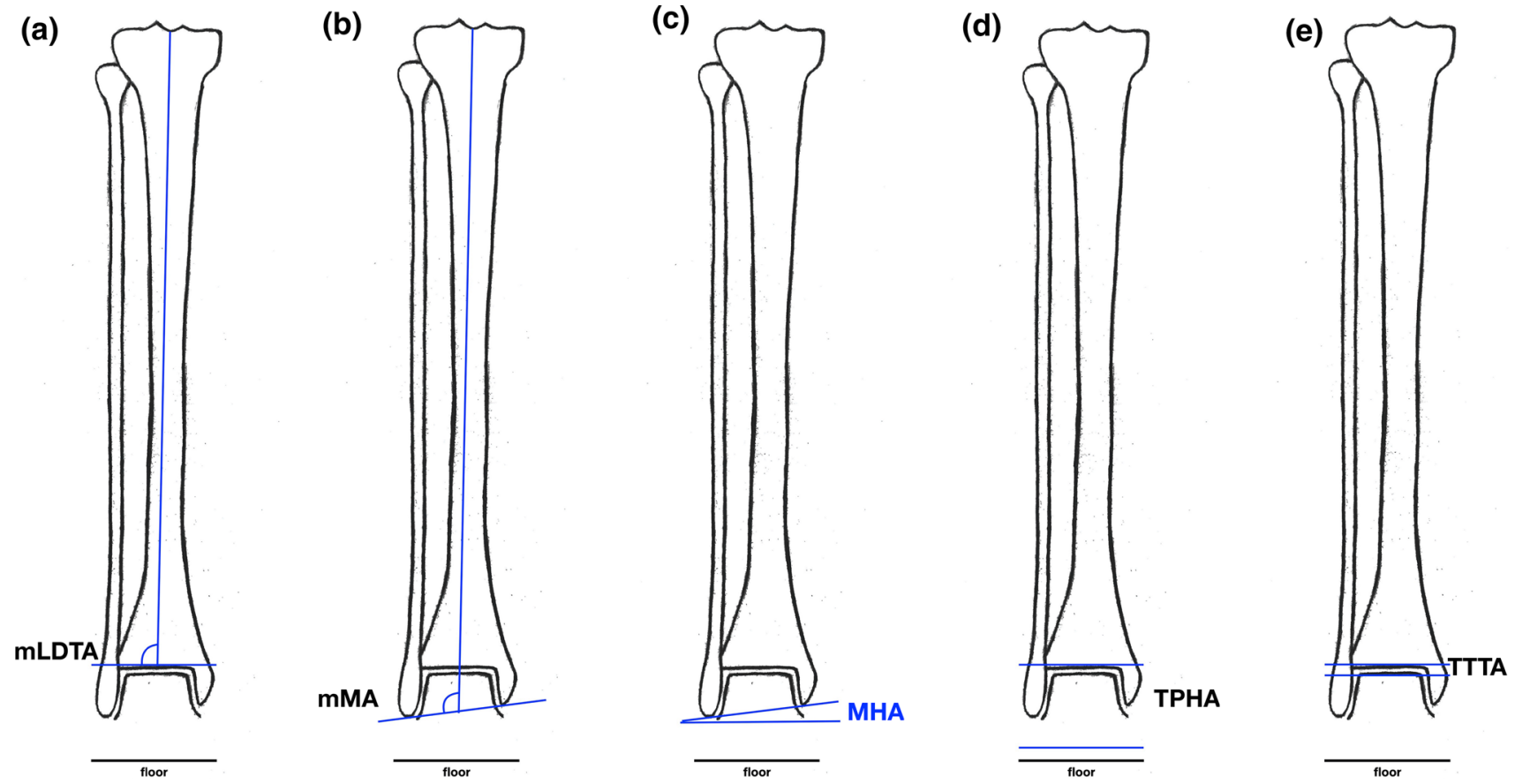

Fig. 4 Illustration of the radiographic parameters measured on a longleg standing X-ray with the knees pointing forward. Measures around the ankle. a mLDTA: angle between tibiaplafond and mechanical tibia axis. $\mathbf{b}$ mMA: angle between malleolar tips and mechanical tibia axis. c MHA: angle between malleolar tips and floor. d TPHA: angle between tibiaplafond and floor. e TTTA: angle between tibio-talar joint surfaces. $m L D T A$, Mechanical Lateral Distal Tibia Angle; $m M A$, Mechanical Malleolar Angle; $M H A$, Malleolar Horizontal Orientation Angle; TPHA, Tibia Plafond Horizontal Orientation Angle; TTTA, Tibio Talar Tilt Angle
Table 1 Radiographic measures in patients undergoing valgisation osteotomies around the knee

\begin{tabular}{lllll}
\hline $\begin{array}{l}\text { Radiographic } \\
\text { measure }\left[{ }^{\circ}\right]\end{array}$ & Preoperative Mean \pm SD (Range) & Postoperative Mean \pm SD (Range) & $\Delta$ & $p$-value \\
\hline HKA & $5.8 \pm 2.9(3.2-17.4)$ & $-0.87 \pm 2.5(-7.0-6.0)$ & -6.7 & $<0.0001$ \\
mMPTA & $85.9 \pm 2.7(77.6-92.9)$ & $90.7 \pm 3.1(77.2-97.3)$ & 4.7 & $<0.0001$ \\
mLDFA & $89.2 \pm 1.8(84.9-94.8)$ & $87.4 \pm 2.0(82.5-92.2)$ & -1.8 & $<0.0001$ \\
mLPFA & $88.8 \pm 9.6(80.0-101.9)$ & $88.9 \pm 4.9(77.9-100.0)$ & 0.13 & 0.011 \\
mLDTA & $87.2 \pm 3.9(76.5-99.8)$ & $85.8 \pm 3.8(76.5-95.2)$ & -1.4 & $<0.0001$ \\
mMA & $101.1 \pm 3.6(90.0-111.0)$ & $100.4 \pm 4.0(86.1-109.2)$ & -0.7 & 0.019 \\
MHA & $16.4 \pm 4.2(3.7-27.8)$ & $10.9 \pm 4.2(-0.2-20.0)$ & -5.5 & $<0.0001$ \\
TPHA & $4.2 \pm 3.4(0.0-5.0)$ & $1.0 \pm 2.9(0.0-2.4)$ & -3.2 & $<0.0001$ \\
TTTA & $0.0(0.0-0.0)$ & $0.0(0.0-0.0)$ & 0.0 & n. s \\
\hline
\end{tabular}

$H K A$, Hip Knee Ankle angle; $m M P T A$, Mechanical Medial Proximal Tibial Angle; $m L D F A$, Mechanical Lateral Distal Femoral Angle; $m L P F A$, Mechanical Lateral Proximal Femoral Angle; $m L D T A$, Mechanical Lateral Distal Tibia Angle; $m M A$, Mechanical Malleolar Angle; $M H A$, Malleolar Horizontal Orientation Angle; TPHA, Tibia Plafond Horizontal Orientation Angle; TTTA, Tibio Talar Tilt Angle demonstrated measuring the HKA, mMPTA/mLDFA, and MHA among other measures as shown in Tables 1 and 2.

High tibial open wedge osteotomy for valgisation of the coronal limb alignment led to a corresponding valgisation of the ankle (Fig. 5). Varisation osteotomies around the knee led to corresponding varisation of the ankle (Fig. 6). 
Table 2 Radiographic measures in patients undergoing varisation osteotomies around the knee

\begin{tabular}{lllll}
\hline $\begin{array}{l}\text { Radiographic } \\
\text { measure }\left[^{\circ}\right]\end{array}$ & Preoperative Mean \pm SD (Range) & Postoperative Mean \pm SD (Range) & $\Delta$ & $p$-value \\
\hline HKA & $-4.3 \pm 3.7(-11.5-5.5)$ & $1.1 \pm 2.2(-3.2-6.7)$ & 5.4 & $<0.0001$ \\
mMPTA & $89.7 \pm 2.9(84.7-96.1)$ & $87.8 \pm 2.6(82.6-92.7)$ & -1.9 & 0.001 \\
mLDFA & $85.7 \pm 2.2(81.5-90.1)$ & $89.3 \pm 2.3(83.6-94.2)$ & 3.6 & $<0.0001$ \\
mLPFA & $87.7 \pm 6.6(71.8-103.1)$ & $87.9 \pm 6.1(74.1-101.9)$ & 0.18 & 0.667 \\
mLDTA & $85.9 \pm 4.5(75.1-93.2)$ & $85.7 \pm 4.9(71.0-93.0)$ & -0.2 & 0.424 \\
mMA & $98.8 \pm 6.5(78.7-111.2)$ & $98.3 \pm 6.4(78.1-104.0)$ & -0.5 & 0.731 \\
MHA & $8.8 \pm 5.1(-2.5-18.5)$ & $11.2 \pm 3.2(4.9-17.0)$ & 2.3 & 0.002 \\
TPHA & $-2.8 \pm 6.2(-13.2-17.7)$ & $0.2 \pm 2.8(-6.2-10.3)$ & 3.0 & $<0.001$ \\
TTTA & $0.0(0.0-0.0)$ & $0.0(0.0-0.0)$ & 0 & n. s \\
\hline
\end{tabular}

HKA, Hip Knee Ankle angle; $m M P T A$, Mechanical Medial Proximal Tibial Angle; $m L D F A$, Mechanical Lateral Distal Femoral Angle; $m L P F A$, Mechanical Lateral Proximal Femoral Angle; $m L D T A$, Mechanical Lateral Distal Tibia Angle; $m M A$, Mechanical Malleolar Angle; MHA, Malleolar Horizontal Orientation Angle; TPHA, Tibia Plafond Horizontal Orientation Angle; TTTA, Tibio Talar Tilt Angle

\section{Discussion}

We determined the effects of osteotomies around the knee on the corresponding frontal alignment of the ankle. The most important findings of this study demonstrate that an osteotomy around the knee for valgisation or varisation of the long leg axis leads to a reorientation of the ankle in the coronal plane. This can be measured using the MHA (Figs. 4, 5, 6, Tables 1, 2).

The main limitation of the study could be seen in the fact that a 3-dimensional reality has been simplified using 2-dimensional radiography. Addressing this problem would require complex imaging in a functional standing position. Given that the aim of this study was to prove the concept in a feasible standard clinical setting, the authors agreed on the sufficiency of the design chosen in this study.

This work was based on the idea that a change of the weight-bearing line at the knee could alter the coronal orientation of the ankle. Given that a long leg standing X-ray is performed with the knees pointing forward, it can be deduced that the X-ray represents a natural illustration of a standing position of the lower extremity [17-20]. Therefore, one must understand that for an even foot sole contact with the ground after valgisation or varisation osteotomy around the knee, the joints distal to the talus (mainly the subtalar joint) have to adapt by inversion or eversion. This alters not only kinematics, but it must also influence the distribution of joint reaction forces [21].

Regarding the ankle joint, unintentional valgisation or varisation might deteriorate biomechanics especially in patients with ligamentous instability. In the study cohort, the TTTA was $0^{\circ}$ in all patients pre- and postoperatively. This could be different and changed by valgisation or varisation in unstable ankle joints, leading to new or aggravated symptoms of instability and pain [19]. In general, unphysiological joint angles should be avoided by meticulous planning and conducting of correction osteotomies [16, 18, 19].

Today, in the era of sub-specialization, communication between the knee surgeon and the foot and ankle surgeon is important. As discussed above, a valgisation or varisation osteotomy around the knee changes the coronal alignment of the ankle, which can be measured using the MHA. The subtalar joint needs to compensate for this with inversion after valgisation osteotomy and eversion after varisation osteotomy in order to secure even foot sole contact with the floor.

This requires a certain mobility in the subtalar joint. Enough range of motion in this region might not be given in patients with an ankle arthrodesis by a nail. In patients with indication for osteotomy around the knee and primary or secondary arthritis of the ankle joints, we would recommend performing the osteotomy around the knee first and then treat the ankle afterwards - especially when indicating ankle arthrodesis [22-27]. With this sequence of surgeries, the surgeon can sustainably accomplish a correct foot position when performing the ankle arthrodesis.

So, not only in symptomatic knees and hips, but also in patients with disease of the ankle, the adjacent joint should be examined and the long-leg axis should be analyzed as part of the routine preoperative workup [28]. 
(a)

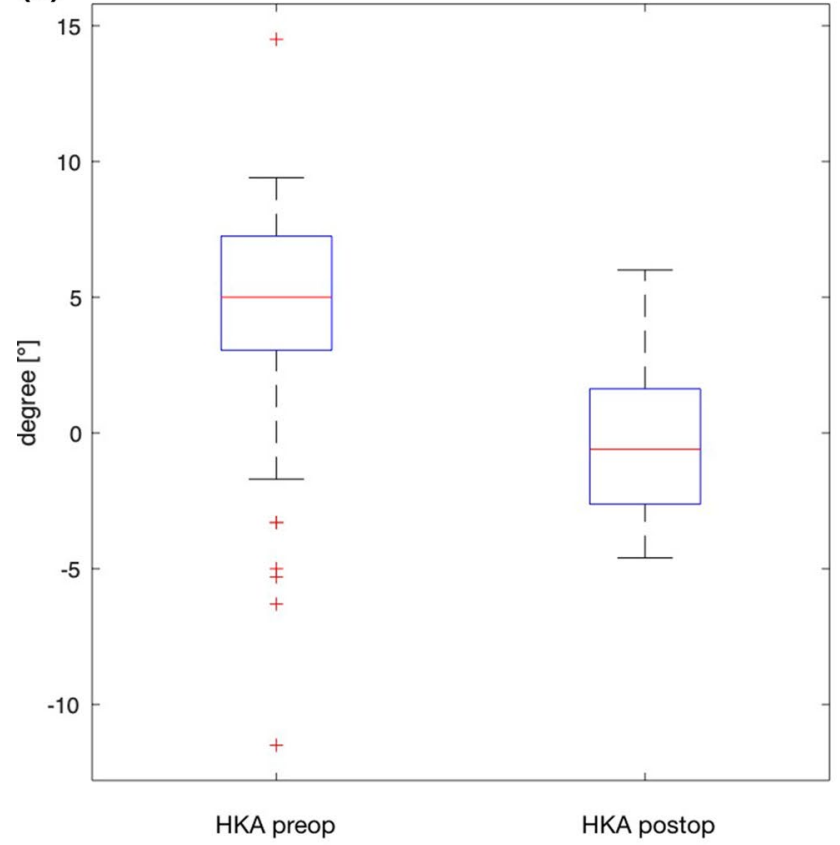

(b)

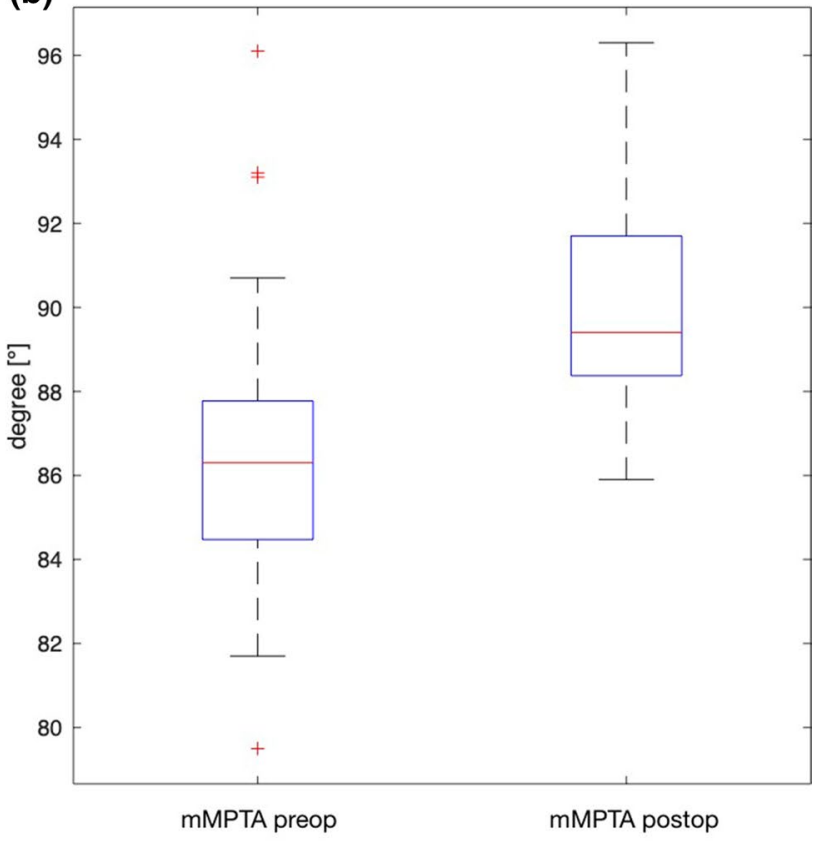

(c)

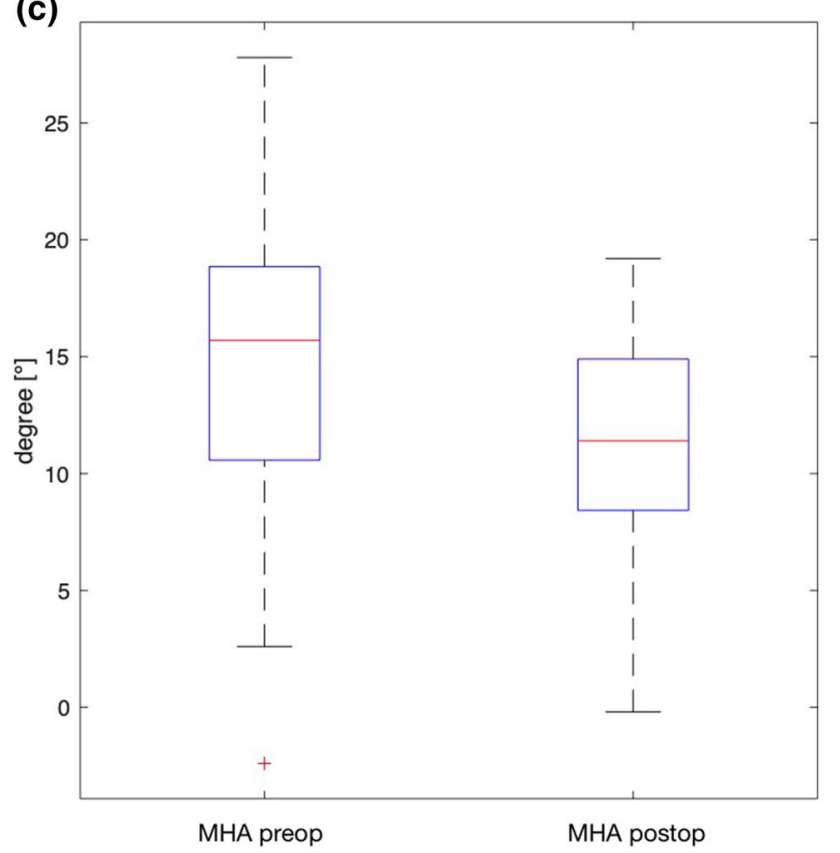

Fig. 5 Open wedge high tibial osteotomy for valgisation. a Mechanical tibio-femoral angle. b Frontal alignment of the proximal tibia. c Coronal alignment of the ankle. HKA, Hip Knee Ankle angle; $M H A$,
Malleolar Horizontal Orientation Angle; $m M P T A$, Mechanical medial proximal tibial angle 

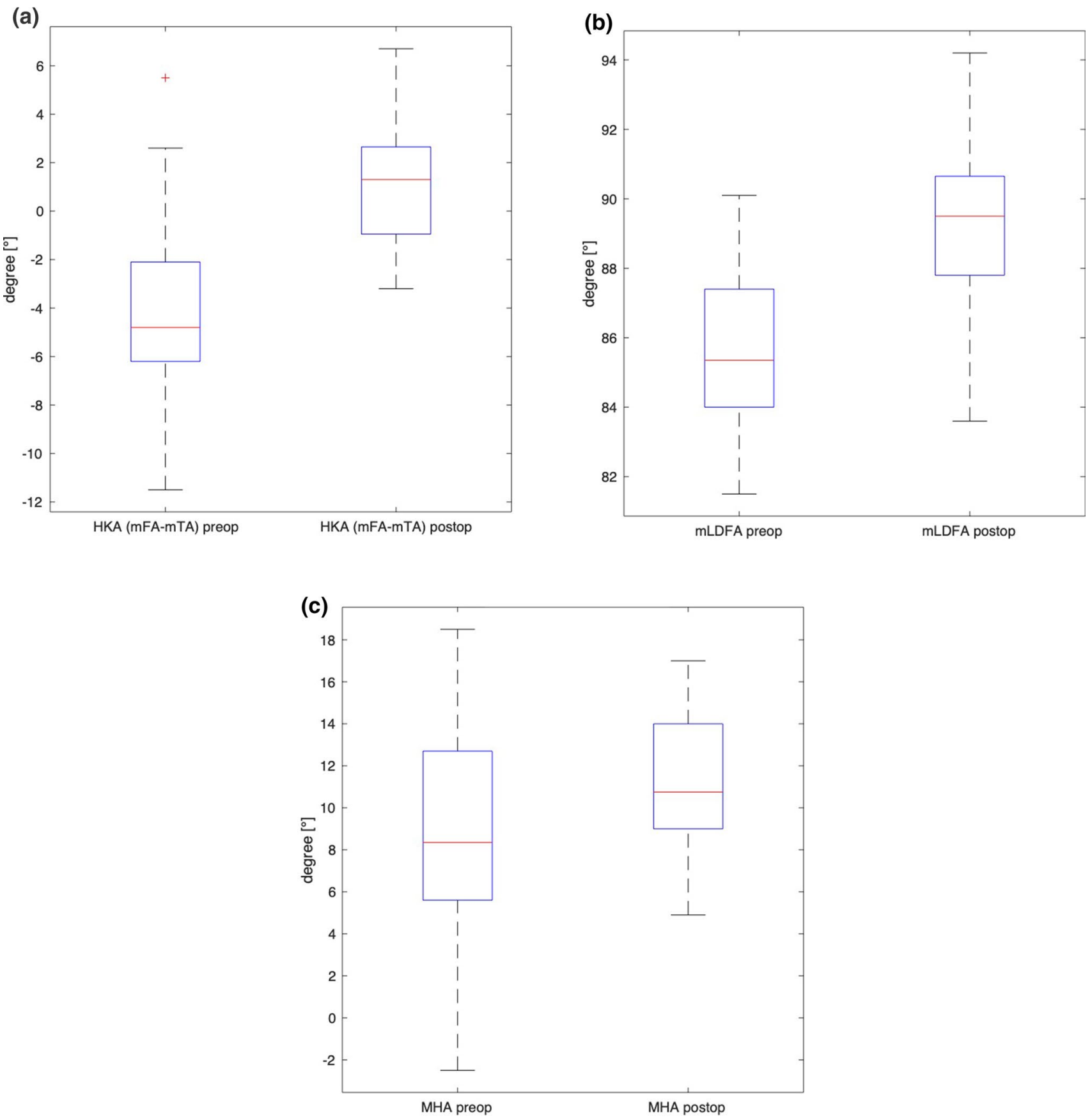

Fig. 6 Varisation osteotomies around the knee. a Mechanical tibio-femoral angle. b Frontal alignment of the distal femur. c Coronal alignment of the ankle. HKA, Hip Knee Ankle angle; $M H A$, Malleolar Horizontal Orientation Angle; $m L D F A$, Mechanical Lateral Distal Femoral Angle

\section{Conclusions}

Osteotomies around the knee for correction of coronal limb alignment not only lead to lateralization or medialization of the weight-bearing line at the knee but also lead to a corresponding coronal reorientation of the ankle. This can be measured at the ankle using the MHA and other modalities. When planning an osteotomy around the knee for correction of genu varum or valgum, the ankle should also be appreciated-especially in patients with preexisting deformities, ligament instabilities, or joint degeneration around the ankle. 
Funding Open Access funding enabled and organized by Projekt DEAL.

Availability of data and material The datasets generated and analyzed during the current study are available from the authors on reasonable request.

\section{Declaration}

Conflict of interest The author declare that they have no conflict of interest.

Open Access This article is licensed under a Creative Commons Attribution 4.0 International License, which permits use, sharing, adaptation, distribution and reproduction in any medium or format, as long as you give appropriate credit to the original author(s) and the source, provide a link to the Creative Commons licence, and indicate if changes were made. The images or other third party material in this article are included in the article's Creative Commons licence, unless indicated otherwise in a credit line to the material. If material is not included in the article's Creative Commons licence and your intended use is not permitted by statutory regulation or exceeds the permitted use, you will need to obtain permission directly from the copyright holder. To view a copy of this licence, visit http://creativecommons.org/licenses/by/4.0/.

\section{References}

1. Kosashvili Y, Safir O, Gross A, Morag G, Lakstein D, Backstein D (2010) Distal femoral varus osteotomy for lateral osteoarthritis of the knee: a minimum ten-year follow-up. Int Orhtop 34:249-254

2. Saragaglia D, Rouchy RC, Krayan A, Refaie R (2014) Return to sports after valgus osteotomy of the knee joint in patients with medial unicompartmental osteoarthritsi. Int Orthop 38:2109-2114

3. Staubli AE, Jacob HAC (2010) Evolution of open-wedge hightibial osteotomy: experience with a special angular stable device for internal fixation without interposition material. Int Orthop 34:67-172

4. Benzakour T, Hefti A, Lemseffer M, El Ahmadi JD, Bouyarmane H, Benzakour A (2010) High tibial osteotomy for medial osteoarthritis of the knee: 15 years follow-up. Int Orthop 34:209-215

5. Wolcott M, Traub S, Efird C (2010) High tibial osteotomies in the young active patient. Int Orthop 34:161-166

6. Amendola A, Bonasia DE (2010) Results of high tibial osteotomy: review of the literature. Int Orthop 34:155-160

7. Paley D, Herzenberg J (2005) Radiographic Assessment of Lower Limb Deformities. In: Principles of Deformity Correction. Springer: Berlin, Heidelberg, New York. pp 31-60.

8. Schröter S, Elson DW, Ateschrang A, Ihle C, Stöckle U, Dickschas J, Harrer J (2017) Lower limb deformity analysis and the planning of an osteotomy. J Knee Surg 30:393-408

9. Staubli AE, De Simoni C, Babst R, Lobenhoffer P (2003) TomoFix: a new LCP-concept for open wedge osteotomy of the medial proximal tibia: early results in 92 cases. Injury 34(Suppl2):B55-62

10. Lobenhoffer P, Agneskirchner JD (2003) Improvements in surgical technique of valgus high tibial osteotomy. Knee Surg Sports Traumatol Arthrosc 11:132-138

11. Lobenhoffer P, Agneskirchner J, Zoch W (2004) Open valgus alignment osteotomy of the proximal tibia with fiation by medial plate fixator. Orthopade 33:153-160

12. Stähelin T, Hardegger F, Ward JC (2000) Supracondylar osteotomy of the femur with use of compression: osteosynthesis with a malleable implant. J Bone Joint Surg Am 82:712-722
13. Visser J, Brinkman JM, Bleys RL, van Heerwaarden CRM, RJ, (2013) The safety and feasibility of a less invasive distal femur closing wedge osteotomy technique: a cadaveric dissection study of the medial aspect of the distal femur. Knee Surg Sports Traumatol Arthrosc 21:220-227

14. Lobenhoffer P, Kley K, Freiling D, van Heerwaarden R (2017) Medial closed wedge osteotomy of the distal femur in biplanar technique and a specific plate fixator. Oper Orthop Traumatol 29:306-319

15. Brinkman J-M, Hurschler C, Agneskirchner J, Lobenhoffer P, Castelein RM, van Heerwaarden RJ (2014) Biomechanical testing of distal femur osteotomy plate fixation techniques. J Exp Orthop $1: 1-7$

16. Schröter S, Nakayama H, Yoshiya S, Stöckle U, Ateschrang A, Gruhn J (2019) Development of the double level osteotomy in severe varus osteoarthritis showed good outcome by preventing oblique loint line. Arch Orthop Trauma Surg 139:519-527

17. Jiang X, Xie K, Han X, Ai S, Wu H, Wang L, Yan M (2020) HKA Angle-A Reliable Planning Parameter for High Tibial Osteotomy: A Theoretical Analysis Using Standing Whole-Leg Radiographs. J Knee Surg. https://doi.org/10.1055/s-0040-1712945

18. Bartholomeeusen S, Van den Bempt M, van Beek N, Claes T, Claes S (2020) Changes in knee joint line orientation after high tibial osteotomy are the result of adaptation of the lower limb to the new alignment. Knee 27:777-786

19. Kim JG, Suh DH, Choi GW, Koo BM, Kim S-G (2020) Change in weight-bearing line ratio of the ankle joint and ankle joint line orientation after knee arthroplasty and high tibial osteotomy in patients with genu varum deformity. Int Orthop 45(1):117-124

20. Kyung MG, Cho YJ, Hwang S, Lee D-O, Lee MC, Lee DY (2021) Change in intersegmental foot and ankle motion after a gigh tibial osteotomy in genu varum patients. J Orthop Res 39(86):93

21. Wang W, Ding R, Zhang N, Hernigou P (2020) Subchondral bone cysts regress after correction of malalignment in knee osteoarthritis: comply with Wolff's law. Int Orthop 45(2):445-451

22. Takeuchi R, Saito T, Koshino T (2008) Clinical results of a valgus high tibial osteotomy for the treatment of osteoarthritis of the knee and the ipsilateral ankle. Knee 15:196-200

23. Choi GW, Yank JH, Park JH, Yun HH, Lee YI, Chae JE, Yoon JR (2017) Changes in coronal alignment of the ankle joint after high tibial osteotomy. Knee Surg Sports Traumatol Arthrosc 25:838-845

24. Choi JY, Song SJ, Kim SJ, Kim SH, Park JS, Suh JS (2018) Changes iin hindfoot alignment after high or low tibial osteotomy. Foot Ankle Int 39:1097-1105

25. Yoshimoto K, Noguchi M, Yamada A, Nasu Y (2019) Compensatory function of the subtalar joint for lower extremity malalignment. Adv Orthop 2019:7656878. https://doi.org/10.1155/2019/ 7656878

26. Graef F, Falk R, Tsitsilonis S, Parka C, Zahn RK, Hommel H (2020) Correction of excessive intraarticular varus deformities in total knee arthroplasty is associated with deteriorated postoperative ankle function. Knee Surg Sports Traumatol Arthrosc 28:3758-3765

27. Graef F, Hommel H, Falk R, Tsitsilonis S, Zahn RK, Perka C (2020) Correction of severe valgus osteoarthritis by total knee arthroplasty is associated with increased postoperative ankle symptoms. Knee Surg Sports Traumatol Arthrosc. https://doi. org/10.1007/s00167-020-06246-4

28. Mounasamy V, Sambandam S (2008) Surgical managementn of young arthritic knee: a review. Eur J Orthop Surg Traumatol $18: 555-564$

Publisher's Note Springer Nature remains neutral with regard to jurisdictional claims in published maps and institutional affiliations. 